\title{
Americium systemic model for rats
}

\author{
Dunstana Melo ${ }^{1}$, Guthrie Miller ${ }^{2}$, John Klumpp ${ }^{3}$, Deepesh Poudel $^{3}$, Waylon Weber ${ }^{4}$, Jasen \\ Swanson $^{5}$, and Raymond Guilmette ${ }^{6}$ \\ ${ }^{1}$ Melohill Technology, LLC, Rockville, MD \\ ${ }^{2}$ Santa Fe, NM \\ ${ }^{3}$ Radiation Protection Division, Los Alamos National Laboratory, Los Alamos, NM \\ ${ }^{4}$ Lovelace Biomedical, Albuquerque, NM \\ ${ }^{5}$ Department of Nuclear Engineering \& Health Physics, Idaho State University, Pocatello, ID \\ ${ }^{6}$ Ray Guilmette and Associates, LLC, Perry, ME 04667, USA
}

\section{Introduction}

Americium-241 is an internal and external radiation hazard often encountered at nuclear reactors and nuclear weapons facilities. It is a man-made metal usually produced by the decay of ${ }^{241} \mathrm{Pu}$. In this work, a baseline compartmental model of the distribution and distribution of americium in rat following a systemic intake is derived. We take a novel pharmacokinetic (PK) front-end modeling approach in which physiological knowledge of vascular flow to the tissues and volumes of extracellular fluid (ECF) associated with the tissues are taken into account. The movement of material into ECF spaces is rapid and is completely characterized by vascular flow rates to and from a tissue and the volumes of the tissue and the ECF associated with the tissue [1]. Other transport, presumably involving cellular processes, is modeled in a purely empirical manner. The PK model incorporates the amount of ECF available in each tissue, which the authors anticipate being useful for chelation modelling. It is also shown to provide a significantly better fit to the data when compared to a more traditional biokinetic model.

\section{Materials and Methods}

The systemic biokinetic model for americium presented here is based on data of distribution and retention of ${ }^{241} \mathrm{Am}$ obtained from studies conducted at Lovelace Respiratory Research Institute (LRRI). Some data of the ${ }^{241} \mathrm{Am}$ distribution in rats have already been published elsewhere [2]; the data are in very good agreement with other publications [3-5]. The data used in the current analysis is the most comprehensive set of data available for ${ }^{241} \mathrm{Am}$ intravenously injected in rats.

The empirical modeling approach is described in Ref. [6]. The model for the animal is broken up into subsystems that interact through a central compartment like plasma or blood, which is fit to the blood data to determine a prescribed function of time plasma(t) or blood(t). Independently for each subsystem, tissues are modeled as sums of a small number of compartments, which interact with the central compartment. The number of 
compartments used is the minimum needed to obtain a satisfactory value of ChiSq/nData (approximately 1) for the subsystem. This is an imprecise concept that involves some judgement. The Plasma(t) [or Blood(t)] method (sometimes referred to as the open-loop method) allows breaking up an unmanageably large model (on the order of 50 unknown parameters) into manageable pieces with simple enough structure to be amenable to trialand-error fitting. Furthermore, this method can be iterated, where the functional form of Plasma(t) or Blood(t) is that obtained by solving the entire model self consistently and then reapplied to individual subsystems. The iterated method allows more accurate Markov Chain Monte Carlo (MCMC) modeling of the smaller-dimensionality subsystems.

\section{Results}

The transfer rates and their uncertainties and correlations were studied using Markov Chain Monte Carlo. Uncertainty is found to be a sensitive function of complexity of the model structure.

Regarding the gross distribution of americium, at 1-hour post IV intake, material has almost entirely left the plasma and relocated to liver (56\% in liver) and bone (20\% in bone). In the PK modeling approach, material goes rapidly to ECF and then transfers to liver (15 min time scale) and skeleton (30 min time scale). The plasma circulation time (from plasma volume divided by cardiac output) is about 16 seconds or $2 \times 10^{-4}$ day, and in the PK model the transfer out of blood is on this time scale. In the PK model this early-time transfer is from blood into ECF, and then later, on a 15-30 min time scale, transfer to liver and bone.

Both the non-PK and PK modelling approaches provide a reasonable description of the dataset, but the PK approach has a significantly larger chain-average likelihood. Either model could be used when modelling the perturbation of americium biokinetics by chelation therapy, but the PK model might be useful in predicting the relative chelation in intra-cellular and extra-cellular fluid spaces.

\section{References}

1. Miller G, Klumpp JA, Melo D, Poudel D. Health phys. 113(6), 519-526

2. Doyle-Eisele M, Weber W, Melo DR, Guilmette RA. International journal of radiation biology. 1;90(11):1068-74. (2014)

3. Durbin, Patricia W. DISTRIBUTION OF TH2 TRANSURANIC ELEMENTS IN MAMMALS. (1962)

4. Taylor DM. Health phys. 8(6):673-7. (1962)

5. Lloyd RD, Mays CW, Taylor GN, Atherton DR. MJ RETENTION AND DOSIMETRY. (1970)

6. Miller G, Bertelli L, Klare K, Weber W, Doyle-Eisele M, Guilmette R. Health phys. 103(4):484-94. (2012) 